\title{
CHEMISTRY IN DENSE MOLECULAR CLOUDS: THEORY AND OBSERVATIONAL CONSTRAINTS
}

\author{
Geoffrey A. Blake \\ Division of Geological and Planetary Sciences \\ California Institute of Technology 170-25 \\ Pasadena, Califomia 91125
}

\begin{abstract}
For the most part, gas phase models of the chemistry of dense molecular clouds predict the abundances of simple species rather well. However, for larger molecules and even for small systems rich in carbon these models often fail spectacularly. We present a brief review of the basic assumptions and results of large scale modeling of the chemistry in dense molecular clouds. Particular attention will be paid to the influence of the gas phase ratios of the major elements in molecular clouds, and the likely role grains play in maintaining these ratios as clouds evolve from initially diffuse objects to denser cores with associated stellar and planetary formation.

Recent spectral line surveys at centimeter and millimeter wavelengths along with selected observations in the submillimeter have now produced an accurate "inventory" of the gas phase elemental budgets in different types of molecular clouds, though gaps in our knowledge clearly remain. The constraints these observations place on theoretical models of interstellar chemistry can be used to gain insights into why the models fail, and show also which neglected processes must be included in more complete analyses. Looking toward the future, truly protostellar regions are only now becoming available for both experimental and theoretical study, and some of the expected modifications of molecular cloud chemistry in these sources are therefore outlined.
\end{abstract}

\section{INTRODUCTION}

In the short span of less than two decades molecular astrophysics, the observational and theoretical examination of molecules in extraterrestrial environments, has grown from a small field of limited importance to one of the most active and innovative branches of many disciplines. The Five College Radio Astronomy Observatory (FCRAO) 14 meter telescope has been particularly prominent in this area, and along with other observatories has literally revolutionized our understanding of the interstellar medium in general, and star formation in particular. An entirely new type of object - the dense molecular cloud - has been discovered, which we now know to be the birthplace of stars, some of them like our own sun. Over ninety atomic and molecular species have been identified in these clouds, a richness and complexity unexpected even a decade ago. Table 1 presents a listing of the currently observed interstellar molecules, and as may be immediately seen the majority of these species contain carbon. Indeed, nearly every functional group in organic chemistry may be found in the interstellar medium!

The dust in molecular clouds renders them opaque at optical and near infrared wavelengths. Because of this the gas and dust in "typical" molecular clouds have temperatures on the order of $10-100 \mathrm{~K}$, and the bulk of their radiation is emitted throughout the millimeter (10-1 mm), submillimeter $(1-0.2 \mathrm{~mm})$, and far-infrared $(200-30 \mu \mathrm{m})$ regions of the spectrum where the inter- 
Simple Inorganic Molecules:

$\begin{array}{llll}\mathrm{H}_{2} & \mathrm{CH}_{4}{ }^{*} & \mathrm{CS} & \mathrm{NaCl}^{*} \\ \mathrm{HCl} & \mathrm{SiH}_{4}{ }^{*} & \mathrm{SiS} & \mathrm{AlCl}^{*} \\ \mathrm{H}_{2} \mathrm{O} & \mathrm{C}_{2} \mathrm{H}_{4}{ }^{*} & \mathrm{OCS} & \mathrm{KCl}^{*} \\ \mathrm{H}_{2} \mathrm{~S} & \mathrm{CO} & \mathrm{SO}_{2} & \mathrm{AlF}^{*} \\ \mathrm{NH}_{3} & \mathrm{SiO} & \mathrm{HNO} ? & \end{array}$

Nitriles, Acetylene Derivatives, \& Related Molecules:

$\begin{array}{llll}\mathrm{PN} & \mathrm{HC}_{3} \mathrm{~N} & \mathrm{CH}_{3} \mathrm{CN} & \mathrm{C}_{2} \mathrm{H}_{3} \mathrm{CN} \\ \mathrm{HCN} & \mathrm{HC}_{5} \mathrm{~N} & \mathrm{CH}_{3} \mathrm{C}_{2} \mathrm{CN} & \mathrm{C}_{2} \mathrm{H}_{5} \mathrm{CN} \\ \mathrm{HNC} & \mathrm{HC}_{7} \mathrm{~N} & \mathrm{CH}_{3} \mathrm{C}_{4} \mathrm{CN} & \mathrm{HNCO} \\ \mathrm{HCCH}^{*} & \mathrm{HC}_{9} & \mathrm{CH}_{3} \mathrm{CCH} & \mathrm{HNCS} \\ \mathrm{CCCO} & \mathrm{HC}_{11}{ }^{\mathrm{N}} & \mathrm{CH}_{3} \mathrm{C}_{4} \mathrm{H} & \mathrm{CCCS}\end{array}$

Aldehydes, Alcohols, Esters, and Amides:

$\begin{array}{llll}\mathrm{H}_{2} \mathrm{CO} & \mathrm{CH}_{3} \mathrm{OH} & \mathrm{HCOCH}_{3} & \mathrm{HCONH}_{2} \\ \mathrm{H}_{2} \mathrm{CS} & \mathrm{CH}_{3} \mathrm{SH} & \mathrm{HCOOH} & \mathrm{H}_{2} \mathrm{CNH} \\ \mathrm{H}_{2} \mathrm{CCO} & \mathrm{CH}_{3} \mathrm{CH}_{2} \mathrm{OH} & \mathrm{HCOOCH}_{3} & \mathrm{H}_{2} \mathrm{NCN} \\ \mathrm{HCOCH}_{3} & \left(\mathrm{CH}_{3}\right)_{2} \mathrm{O} ? & \mathrm{HCOC}_{2} \mathrm{H}_{2} ? & \mathrm{CH}_{3} \mathrm{NH}_{2}\end{array}$

Cyclic Molecules:

$\mathrm{C}_{3} \mathrm{H}$
$\mathrm{C}_{3} \mathrm{H}_{2}$
$\mathrm{SiC}_{2}$

$\frac{\text { Ions: }}{\mathrm{CH}^{+}}$

$\mathrm{H}_{2} \mathrm{D}^{+}$?

$\mathrm{H}_{3} \mathrm{O}^{+}$?

$\mathrm{HCO}^{+}$

$\mathrm{HOC}^{+}$?

$\mathrm{HN}_{2}{ }^{+}$

$\mathrm{HOCO}^{+}$

$\mathrm{HCNH}^{+}$

$\mathrm{HCS}^{+}$

$\mathrm{SO}^{+}$

Radicals:

$\begin{array}{llll}\mathrm{CH} & \mathrm{C}_{3} \mathrm{H} & \mathrm{CN} & \mathrm{CCS} \\ \mathrm{OH} & \mathrm{C}_{4} \mathrm{H} & \mathrm{C}_{3} \mathrm{~N} & \text { SO } \\ \mathrm{HCO} & \mathrm{C}_{5} \mathrm{H} & \mathrm{NO} & \\ \mathrm{CCH} & \mathrm{C}_{6} \mathrm{H} & \mathrm{NS} & \end{array}$

* Detected only in the envelope around IRC+10216. ?Claimed but not yet confirmed. 
action of electromagnetic radiation with the rotational motion of simple molecules and with the vibration-rotation interactions of larger molecules is at a maximum. The millimeter and submillimeter bands are therefore key windows to the universe, as the composite spectrum in Figure 1 demonstrates. At longer wavelengths the rotational envelopes of heavy molecules dominate the spectra of dense molecular clouds, but in the submillimeter region the lowest transitions of molecular hydrides, the fine structure transitions of low $\mathrm{Z}$ atoms, and the lowest vibrational transitions of long chain hydrocarbons begin to appear.

In addition to producing some of the most accurate estimates of the chemical abundances, temperatures, densities, and velocity structures within molecular clouds, millimeter and submillimeter-wave spectral lines also contribute substantially to the energy balance in these objects. The amount of energy released by rotational and fine structure emission determines, in part, the rates of collapse and ultimately the efficiency of star formation in molecular clouds, and is very sensitive to the exact chemical composition of the gas and dust.

Observations over the last several years have established similarities in the chemical composition of many molecular clouds, but several fundamental problems, such as the large differences in the abundances of key species, remain. For example, it is not known whether the observed differences between the chemical composition of giant molecular cloud complexes and smaller dark clouds, or between dark clouds themselves, is purely a result of the different physical conditions within these objects or whether evolutionary pattems exist.

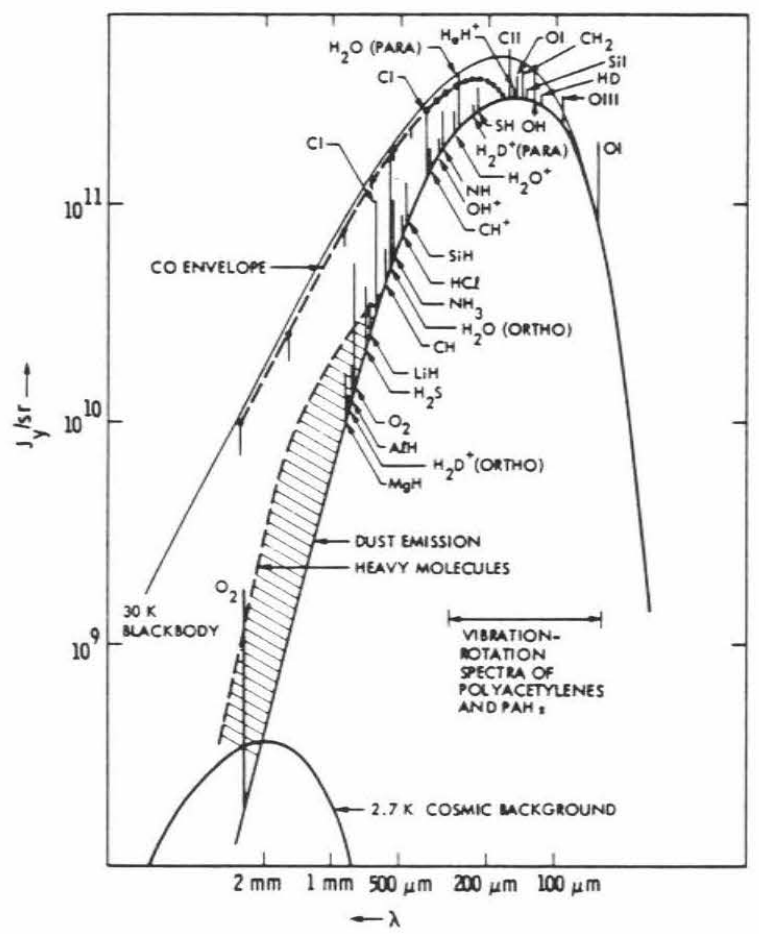

Figure 1.- Composite spectrum of a $30 \mathrm{~K}$ molecular cloud. Only a few of the most fundamental of the host of spectral features have been included. 
Considerable progress has also been made theoretically since the first discovery of complex organic molecules in interstellar space. Several different hypotheses about the nature of chemical synthesis in the interstellar medium have been brought forward, and have been shown to contribute to the extraordinary richness observed. Models of the gas phase and surface chemical reactivity of matter in non-terrestrial environments have become commonplace. As with observational work, however, basic questions still surround the formation of molecular species in the galaxy.

We shall attempt in this paper to outline what is currently known both theoretically and observationally about the formation of complex molecules in dense interstellar clouds and their possible relation to material found in primitive relics of the presolar nebula. Theory is briefly outlined to make this paper as self-contained as possible, and also since no other description of the recent progress made in theoretical work is included in these proceedings. For fuller explanations of the details of various processes outlined here, more comprehensive reviews of molecular cloud chemistry by Herbst ${ }^{1}$ and Prasad et al. ${ }^{2}$, and observational aspects of molecular astrophysics by Irvine et $\mathrm{al}^{3}{ }^{3}$, are recommended. The chemistry within shocks and within outflows from evolved stars can also contribute to the observed chemical and isotopic composition of dense molecular clouds, but shall not be explicitly addressed in this review. A tutorial style will be used throughout, with illustrative, but not comprehensive, references.

\section{CHEMICAL PROCESSES IN THE INTERSTELLAR MEDIUM}

The often harsh environment of interstellar space led early investigators of the chemical content of the interstellar medium to conclude that the gas would be composed entirely of atoms, throughout which was dispersed a family of small $(\leq 0.1 \mu \mathrm{m})$ dust grains. Even now, our understanding of the processing of material from atomic to more complex species in space is hindered by a lack of experimental data. Just what sort of processes are important in molecular clouds? Even in the densest cloud cores, the density is sufficiently low that only bimolecular reactions need be considered in the gas phase; any intermediate complex has sufficient time to react or break up before a third body can carry away excess energy. Temperatures are sufficiently low in most regions of dense molecular clouds to preclude any reactions with even small activation barriers. Gas phase modeling may therefore be confined principally to exothermic binary reactions. Reactions on grain surfaces may also be invoked, although little experimental data is available on the catalytic potential of cosmochemical surfaces at relevant temperatures. Thus, there are really two separate camps: Gas phase processing and catalysis on grain surfaces. Each camp has its own set of defenders and detractors, and, unfortunately, little collaborative work has so far been accomplished. The importance of each to the overall chemistry in dense molecular clouds and in protostellar nebula are beyond question, however.

For example, molecular hydrogen is known to be the dominant form of hydrogen in the cores of dense molecular clouds, yet no adequate scheme for its formation in the gas phase has been found. Observational limits as to the required formation efficiencies on grain surfaces are high, but not excessive ${ }^{4}$. The importance of $\mathrm{H}_{2}$ bond formation to the subsequent chemistry cannot be overestimated, for it is this initial molecular linkage which breaks the bottleneck to the further synthesis of complex species. Conversely, the abundances of molecular ions such as $\mathrm{HCO}^{+}$, of highly unsaturated polyacetylene chains such as $\mathrm{C}_{4} \mathrm{H}$, and certain isomeric behavior in dense clouds are quite difficult to reconcile with formation on grain surfaces, but are quite well predicted by gas phase models of interstellar chemistry incorporating ion-molecule reactions. Relevant aspects of gas phase chemistry are better understood at present, and we begin accordingly with a discussion of what has been discovered about such processes in dense molecular clouds. 


\section{Gas Phase Chemistry in Dense Clouds}

As noted above, the low densities and temperatures in dense molecular clouds restricts viable gas phase mechanisms to exothermic reactions without activation barriers. Except for certain radical-radical reactions, nearly all exothermic reactions between neutral species possess substantial activation energies and may be safely neglected. Ion-molecule reactions, on the other hand, often proceed at the collision rate - any activation barrier is overcome by the gain in kinetic energy of the collision complex as reaction occurs ${ }^{5}$. The long range nature of the ion/induced dipole force also creates significantly larger rate constants than those for even the fastest neutralneutral reactions. Further, it has recently been discovered that certain exothermic ion-molecule reaction rates involving highly polar species increase dramatically at lower temperatures due to dipole locking and tunneling effects ${ }^{6}$. All of these factors combine to make ion-molecule reactions the dominant driver of gas phase chemistry in cold, dense clouds.

Many different types of reactions are important to molecular cloud chemistry. These include purely chemical processes such as hydrogenation, proton transfer, condensation, electron recombination, fixation, etc. in which particles are used to carry away the excess reaction energy; and radiative association reactions in which the collision complex emits radiation to stabilize itself. All of these reactions, and several radical-radical steps, are essential to the formation of the pivotal $\mathrm{C}, \mathrm{N}$, and $\mathrm{O}$ containing molecules in dense clouds, namely $\mathrm{H}_{2} \mathrm{O}, \mathrm{CO}$, and $\mathrm{N}_{2}$ :

$\mathrm{H}_{2} \mathrm{O}$ :

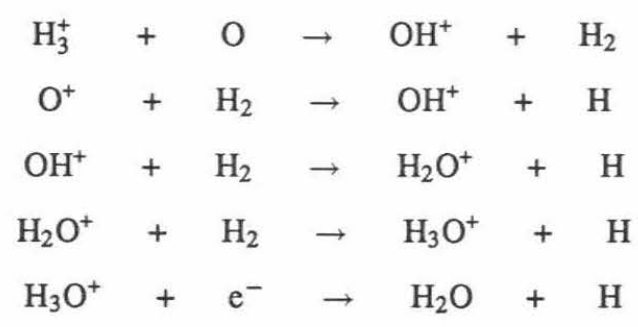

CO:

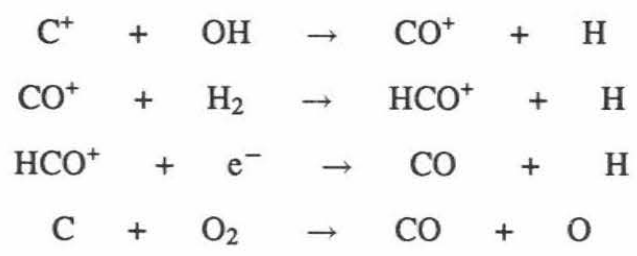

$\mathrm{N}_{2}$ :

$$
\begin{aligned}
\mathrm{NH}_{3}^{+}+\mathrm{e}^{-} & \rightarrow \mathrm{NH}+\mathrm{H}_{2} \\
\mathrm{NH} & +\mathrm{N}
\end{aligned}
$$

on so on. Radiative association reactions are especially important for the production of larger molecules, as we shall see later.

One of the most important aspects of homogeneous gas phase chemistry is the ease with which it may be modeled theoretically. A glance at the many reactive species listed in Table 1, and the high degree of unsaturation found despite the overwhelming $\mathrm{H}_{2}$ abundance, drives home 
the essential point of interstellar chemistry - kinetic factors dominate thermodynamics. The result is a highly non-equilibrium chemical composition that is very sensitive to physical properties of the environment, time scales, and initial conditions. Once appropriate rate constants have been measured or estimated, however, large scale kinetic modeling of the chemistry is possible. The temporal behavior of the concentration $\mathrm{X}$ of a chemical species in the gas phase is given by the sum of three terms:

$$
\left(\mathrm{dX}_{\mathrm{k}} / \mathrm{dt}\right)_{\mathrm{t}}=\left(\mathrm{dX}_{\mathrm{k}} / \mathrm{dt}\right)_{\mathrm{g}}+\left(\mathrm{dX}_{\mathrm{k}} / \mathrm{dt}\right)_{\mathrm{p}}+\left(\mathrm{dX}_{\mathrm{k}} / \mathrm{dt}\right)_{\mathrm{d}} \mathrm{k}=1, \ldots, \mathrm{s}
$$

where

$(\mathrm{dX} / \mathrm{dt})_{\mathrm{g}}=$ rate of change due to gas phase reactions

$\left(\mathrm{dX}_{\mathrm{k}} / \mathrm{dt}\right)_{\mathrm{p}}=$ rate of change due to physical motions (collapse, outflow)

$\left(\mathrm{dX}_{\mathrm{k}} / \mathrm{dt}\right)_{\mathrm{d}}=$ rate of change due to interaction with dust grains

$\mathrm{s}=$ total number of chemical species.

Mathematically, the chemistry is modeled using matrix methods and a master equation for the total rate of change of the concentration of a species $\mathrm{X}_{\mathrm{k}}$, namely

$$
\left(\mathrm{dX}_{\mathrm{k}} / \mathrm{dt}\right)=\sum_{\mathrm{j}=1}^{\mathrm{q}} \nu_{\mathrm{ij}}\left(\alpha_{\mathrm{j}} \prod_{\mathrm{i}=1}^{\mathrm{s}} \mathrm{X}_{\mathrm{i}}^{\mathrm{m}_{\mathrm{ij}}}\right) \quad \mathrm{k}=1, \ldots, \mathrm{s}
$$

over all $i, j . \quad \nu_{\mathrm{ij}}$ are the stochiometric coefficients of a given reaction channel, and $\alpha_{\mathrm{j}}$ are the reaction rates. The resulting equations are stiff, that is the rates of change $\left(\mathrm{dX}_{\mathrm{k}} / \mathrm{dt}\right)$ vary by orders of magnitude, necessitating involved differential equation solvers such as the Gear's method. Nevertheless, modem supercomputer technology allows time dependent calculations with as many as several hundred reactants and several thousand reactions to be performed.

Initial conditions for the differential equations are selected from observations of diffuse clouds, with incremental numerical integration of the total reaction matrix producing the abundances of selected species as a function of time. The most recent calculations have included cosmic ray ionization, positive and negative ion-molecule reactions, radiative association, electron recombination, photoprocessing, etc. Some models incorporating crude dynamical effects have also been reported, but none of these large time dependent models have addressed, in a realistic way, the nature of gas-grain interactions ${ }^{7,8}$. Another major limitation of gas phase modeling is a lack of experimental measurements of some crucial reaction rates and their branching ratios, most notably those involving the neutral atomic species $\mathrm{C}, \mathrm{N}$, and $\mathrm{O}$, and electron recombination processes involving molecular ions.

A simplified view of the ion-molecule chemistry in dense molecular clouds as determined by such large scale models is shown in Figure $2^{9}$. In diffuse clouds, the vast majority of the gas phase carbon is present as $\mathrm{C}^{+}$( $\mathrm{C} \mathrm{II}$ ) while nitrogen and oxygen exist primarily as neutral atoms. In denser clouds, the UV flux is reduced markedly, and impact by cosmic rays becomes the primary ionization mechanism for cloud column densities up to $10^{25} \mathrm{~cm}^{-2}$. At higher column densities the cosmic rays no longer penetrate to the center of the cloud. Initial products of the cosmic ray bombardment are energetic $\mathrm{H}_{2}{ }^{+}$and $\mathrm{He}^{+}$ions. $\mathrm{H}_{2}{ }^{+}$then reacts upon every collision with neutral molecular hydrogen to produce $\mathrm{H}_{3}{ }^{+}$, which along with $\mathrm{He}^{+}$begins to a wide variety of reaction paths among the first and second row elements. In this way, the energy deposited within the cloud by the cosmic rays is converted into chemical complexity, thereby providing the continual input required to maintain the chemical disequilibrium. 


\section{DENSE CLOUDS: \\ FLOW OF IONIZATION}

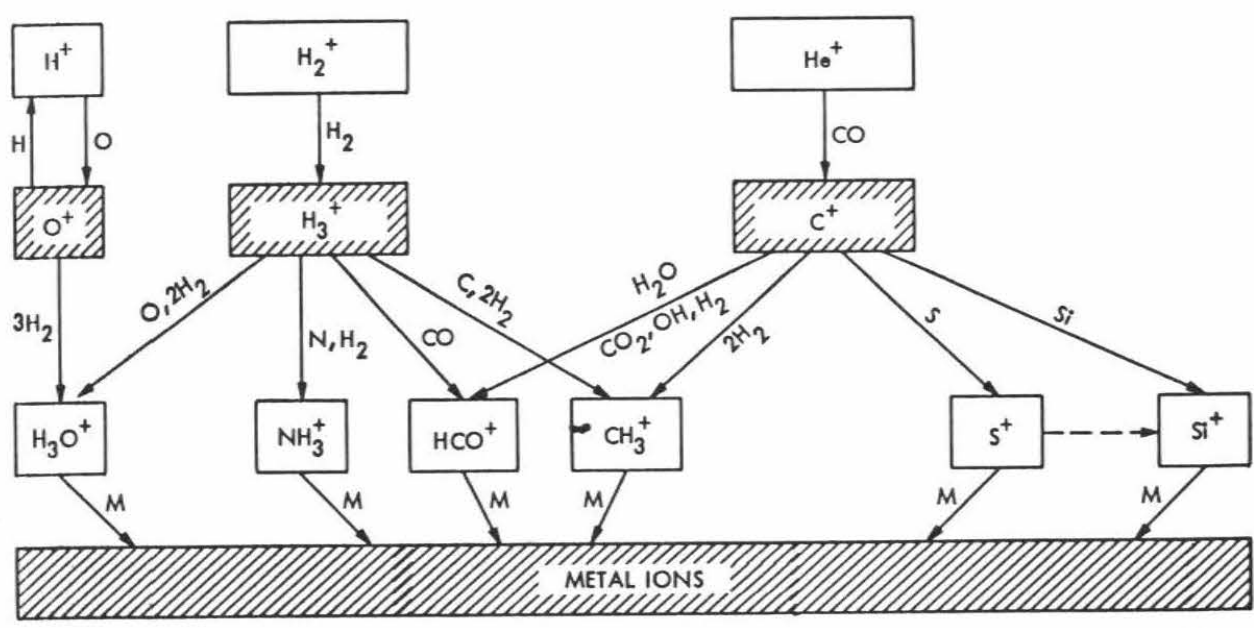

Figure 2.- Flow of ionization in dense clouds and the initiation of gas phase chemistry ${ }^{9}$.

The lack of intense UV sources within most molecular clouds and the large attenuation caused by dust leads to a dramatic drop in the ionization fraction in dense clouds as compared with more diffuse objects. Whereas in diffuse clouds the fractional ionization is near $10^{-4}$ (from the photoionization of atomic carbon), in dense clouds it is closer to $10^{-8}$. Accordingly, the ionized carbon becomes gradually neutralized. Reactions of C II and C I with species such as O, $\mathrm{O}_{2}$, or $\mathrm{OH}$ then form $\mathrm{CO}$, while reactions with $\mathrm{H}_{3}{ }^{+}$or $\mathrm{H}_{2}$ begin the hydrocarbon chemistry. The great stability of $\mathrm{CO}$ is such that this channel dominates over the hydrocarbon routes. Indeed, the only important chemical reaction of $\mathrm{CO}$ is with cosmic ray induced $\mathrm{He}^{+}$, which regenerates $\mathrm{C}^{+}$. Without this "leak", the C II $\rightarrow$ C I $\rightarrow$ CO conversion sequence would completely dominate the carbon chemistry in dense clouds. For example, even when material is driven into the hydrocarbon channels, oxidation still remains the primary limitation to the evolution of molecular complexity. The close coupling of complex molecules with the atomic carbon/CO network is most clear in time dependent calculations of the carbon chemistry, where the C I abundance climbs early in the cloud lifetime but then drops as steady state is approached. Similar curves are observed for more complex species such as $\mathrm{CH}_{3} \mathrm{OH}$, but with a considerable time lag ${ }^{1}$.

Once removed from the $\mathrm{CO}$ cycle, small hydrocarbon ions may participate in a wide variety of reactions leading to complex organic species containing several different functional groups, although the total abundance of such species is only a hundredth of the CO concentration. Coupling of the hydrocarbon chemistry with oxygen leads to alcohols, ethers, and carboxylic acids, while coupling with nitrogen leads to nitriles, amines, and amide linkages. It is with these species that we shall be most concemed, for although they do not comprise a majority of the carbon budget in dense clouds, their formation and abundance depend critically on important cloud parameters such as the oxygen fugacity, depletion, density gradients, shocks, and so on. They may also provide a natural source for some, or most, of the organic material discovered in extraterrestrial media such as Brownlee particles and comets. 
Chemistry of the nitrogen and oxygen families is also initiated by ion-molecule reactions with $\mathrm{H}_{2}$ and $\mathrm{H}_{3}{ }^{+}$to produce small hydrogenated ions, which then couple closely with the carbon cycle primarily via reactions of the neutral atomic species $\mathrm{C}, \mathrm{N}$, and O. Sulfur and silicon chemistry, on the other hand, appears to be initiated and maintained primarily by radical-radical reactions. The difficulty of measuring the reaction rates of such processes and of theoretically predicting the reaction products has resulted in a poor understanding of the gas phase chemistry of these elements. As described below, it is likely that interstellar grains contain a large fraction $(>50 \%)$ of even the most volatile first and second row elements in dense clouds. Nevertheless, certain unique gas phase processes, such as fractionation, make analyses of low temperature ionmolecule and radical-radical chemistry central to an understanding of the formation of not only stars, but their attendant cometary and planetary bodies as well. A more detailed comparison of the predictions of gas phase chemical models with observations is presented later.

\section{Gas-Grain Interactions and Grain Mantle Chemistry}

Even more so than their gas phase counterparts, models of gas-grain interactions and the chemistry which occurs on grain surfaces in cold clouds are plagued by a lack of hard experimental data. This, and the difficulties of quantitatively describing heterogeneous catalysis on surfaces of poorly known composition, has lead, unfortunately, to a neglect of such processes in large scale kinetic modeling of interstellar chemistry beyond the formation of the intial $\mathrm{H}_{2}$ bond. Nevertheless, it is clear from numerous experiments, principally those performed by Greenberg and co-workers ${ }^{10,11}$, that grains will not be chemically inert in the interstellar medium.

Even if grains were chemically inert, however, they would still influence the chemical composition of molecular clouds through selective depletion processes. Indeed, following diffuse cloud observations gas phase models of dense cloud chemistry assume that $80-90 \%$ of the cosmically available $\mathrm{C}, \mathrm{N}$, and $\mathrm{O}$ atoms are depleted onto grains. "Metals" such as $\mathrm{Si}, \mathrm{S}$, or Fe are depleted by factors exceeding 100 . To see why, consider a molecular cloud with a central density of $10^{5} \mathrm{~cm}^{-3}$ and a kinetic temperature of $20 \mathrm{~K}$ with a "standard" gas-to-dust ratio. Simple molecular kinetic calculations show that the time required for complete depletion of the condensible gas phase material onto grains to be

$$
\tau_{\text {accretion }}=10^{9} / \mathrm{n}_{\mathrm{H}_{2}} \mathrm{y}_{\mathrm{s}} \quad \mathrm{yr},
$$

where $y_{s}$ is the sticking coefficient, or about $10^{5}$ years even for models where only one collision in ten results in adsorption. Direct evidence of molecules trapped on grains is provided through the observations of solid state vibrational features of $\mathrm{CO}, \mathrm{H}_{2} \mathrm{O}, \mathrm{NH}_{3}$, etc. in the mid-infrared ${ }^{12}$. This depletion time scale is much less than estimated cloud lifetimes of nearly $10^{7}$ years, and implies that at the very least some efficient desorption mechanism must exist for returning grain mantles into the gas phase. Several such mechanisms have been suggested, ranging from shocks, spot heating by cosmic rays and X-rays, or grain-grain collisions to grain mantle chemical explosions, but none have been definitively established ${ }^{13,14}$.

Estimates of the chemical reactivity of grains must include several processes: collisional capture of gas phase atoms and molecules, migration of trapped species over the grain surface, reaction between species on the grain surface (including photo-processing of the grain mantle) and ejection of reaction products into the gas phase. All of these factors are very sensitive to the exact nature of the grain surface, and may vary widely depending on the reacting species and the substrate. Nevertheless, certain general features have become clear from recent experiments. 
For a more complete review of these processes and their observational consequences, see the contribution from R. Knacke in these proceedings. Basically, it has been determined theoretically that even at the low temperatures characterizing interstellar grains, $\mathrm{H}$ and $\mathrm{H}_{2}$ (and some heavier atoms as well) can "scan" the grain surface rapidly and react with heavier or more strongly bound species. This produces primarily heavily hydrogenated species such as $\mathrm{NH}_{3}, \mathrm{H}_{2} \mathrm{CO}, \mathrm{CH}_{3} \mathrm{OH}$, etc. Exposure of such an icy grain mantle to UV radiation creates energetic radicals within the matrix, which when heated sufficiently can explosively drive off much of the mantle leaving behind an organic refractory residue. In this way, molecules are returned to the gas phase while organic grain mantles continue to grow. The only drawbacks of these otherwise elegant experiments are the difficulty of simulating hydrogen rich grain mantles, and the lack of detailed kinetic data necessary to produce realistic models of the chemical potential of grains in molecular clouds.

As previously noted, models to date have really concentrated on either only gas phase or surface processes. That is to say, large models of the gas phase chemistry have not dealt with depletion onto and chemical modification within grain mantles, and grain mantle models have not incorporated realistic models of the gas phase chemistry. A limited set of calculations by d'Hendecourt et al. ${ }^{15}$ have shown, however, that plausible desorption mechanisms can prevent a complete removal of the gas phase component of dense molecular clouds. In this calculation, the abundances of a small set of molecules, constrained to be identical in the gas phase and in grain mantles for computational stability, were examined using grain-grain collisions and subsequent chemical explosions as the desorption scheme. The requirements for computational stability also underline a second problem with comparing the relative importance of gas phase and surface chemistry to observations, namely, the similarity of simple molecules produced. For example, both gas phase and grain chemistry can produce the observed quantities of $\mathrm{H}_{2} \mathrm{O}, \mathrm{CH}_{3} \mathrm{OH}, \mathrm{HCN}$, etc., and it is not possible based on the observations of such species to conclude which process, if either, dominates. The real key is to find "signposts" of each which may be unambiguously assigned to either process given large observational uncertainties. Molecular ions and reactive isomers like $\mathrm{HNC}$ or $\mathrm{CH}_{3} \mathrm{NC}$ are probably such molecules for gas phase chemistry, neither can be convincingly produced on grain surfaces. Likewise, the observations of significant enhancements of saturated molecules such as $\mathrm{C}_{2} \mathrm{H}_{3} \mathrm{CN}$ or $\mathrm{C}_{2} \mathrm{H}_{5} \mathrm{CN}$ in the warmest regions of active star forming clouds (see next section) are powerful arguments for the pervasiveness of surface catalysis. The most pressing need in grain chemistry models at the moment is experimental measurement of the mobility and reactivity of simple molecules on realistic cosmochemical substrates. We note that such measurements are now feasible using state-of-the-art surface science instruments.

\section{OBSERVATIONAL CHARACTERIZATION OF DENSE CLOUD CHEMISTRY}

Due to the opaque nature of the atmosphere in many wavelength regions and the relatively insensitive nature of early receiver/telescope combinations, most of the previous observational work on the chemistry of dense molecular clouds has proceeded through a biased selection of molecules on a line-by-line basis. Nevertheless, a marvelous pattern of great chemical complexity has emerged from these observations, with both familiar species such as $\mathrm{CO}, \mathrm{H}_{2} \mathrm{O}$, or $\mathrm{CH}_{3} \mathrm{OH}$ and highly unusual molecules like $\mathrm{HCO}^{+}, \mathrm{HC}_{11} \mathrm{~N}$, or $\mathrm{SiC}_{2}$ present in considerable abundance. Although most of the detected molecules have low lying transitions in the microwave range, for accurate measurements of their excitation and abundance it is necessary to examine higher J levels whose transitions occur most prominently in the millimeter and submillimeter regimes. Indeed, the rotational envelope maxima of most molecules in dense clouds lie between 200 and $600 \mathrm{GHz}$ where only a small amount of research has been performed. 

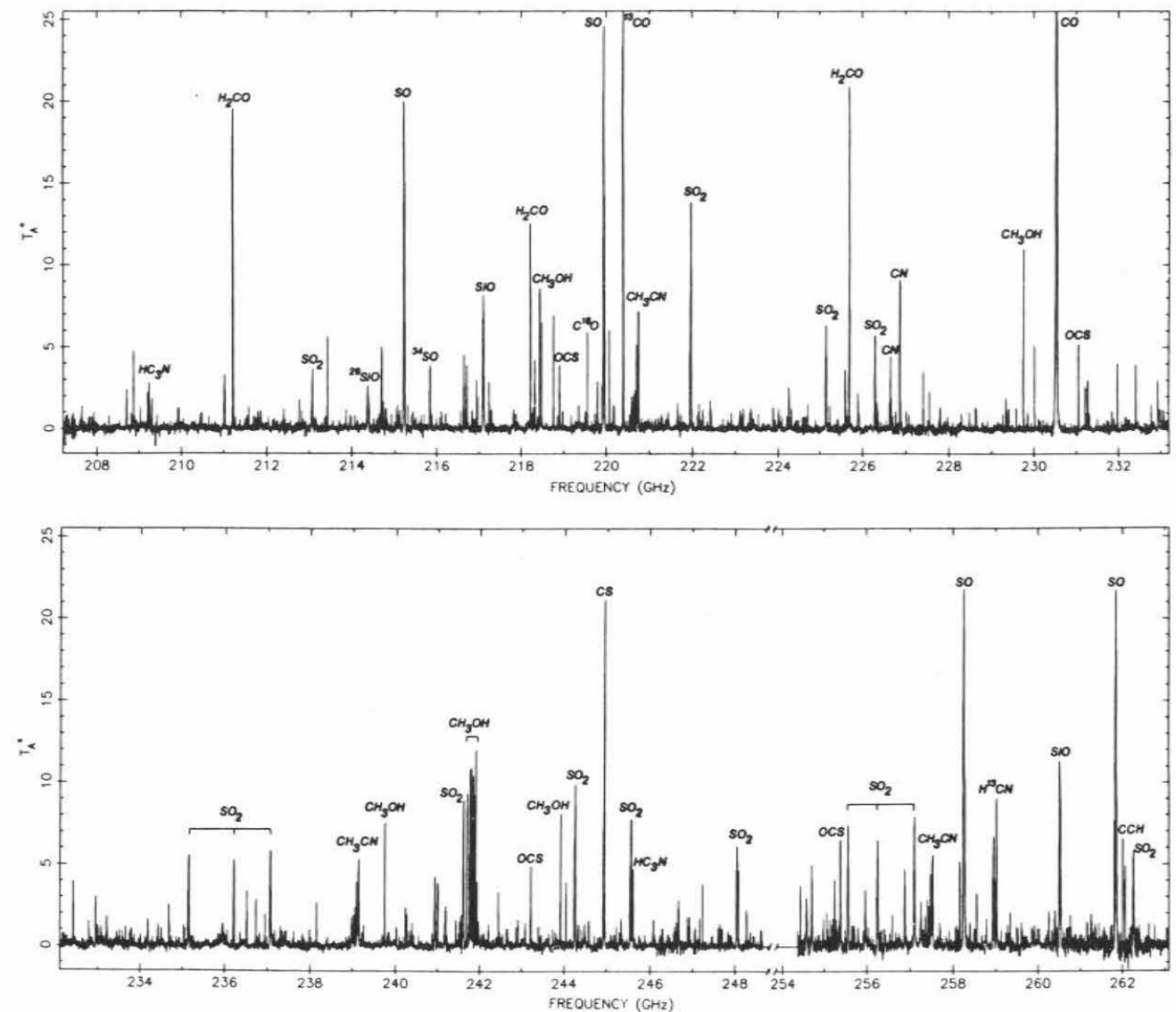

Figure 3.- Compressed millimeter-wave survey of OMC-1 near $1.3 \mathrm{~mm}$. Nearly 900 resolved features have been detected, all but 13 of which are identified.

Recent developments in receiver and telescope technology have allowed unbiased surveys of small regions in the millimeter-wave spectrum below $200 \mathrm{GHz}$ to be recorded for the brightest objects in the sky such as the Orion and Sagittarius giant molecular clouds at the Onsala Radio Observatory and at AT\&T Bell Laboratories ${ }^{16,17}$. Advantages of line surveys versus selected observations include completeness, better calibration, certainty of identification, and measurement of the total integrated line flux. The construction of higher frequency superconductor-insulatorsuperconductor (SIS) receivers at Caltech, and the improved performance possible with the large $10.4 \mathrm{~m}$ telescopes of the Owens Valley Radio Observatory (OVRO), has enabled the completion of a high sensitivity survey of the Orion molecular cloud (OMC-1) between $208-263 \mathrm{GHz}^{18,19}$. This survey was conducted, in part, as an attempt to preview the millimeter and submillimeter wave spectra of dense molecular clouds obtainable with the new generation of submillimeter telescopes now becoming operational worldwide. A compressed view of the spectrum is presented in Figure 3. Nearly 900 resolved spectral features, arising from some thirty species in sixteen varying isotopic substitutions, have been detected. Of these, less than 15 remain currently unidentified! Analyses of the intensities of the emission features after a deconvolution of their line profiles have 
resulted in accurate estimates of the abundances of the molecular species in different regions of the cloud, and have shown that the tremendously heterogeneous composition of the Orion cloud core may be simply interpreted in the framework of the interaction between a quiescent molecular cloud chemistry and that induced by massive star formation within the cloud core. At the noise level of the OVRO survey $(0.2 \mathrm{~K})$ roughly $50 \%$ of the $1.3 \mathrm{~mm}$ spectrum contains detectable emission, with the integrated molecular line flux accounting for at least $40 \%$ of the total radiative output of the cloud at these wavelengths.

The total observing time required for this spectrum was approximately 28 nights during which the average optical depth at the zenith was 0.3. Data reduction took several hundred hours of computer time and months of human analysis. Indeed, identifications of the many lines left after comparison with publicly available spectral line catalogs would not have been possible without a vigorous, complementary laboratory program carried out at Duke University and the Jet Propulsion Laboratory. Catalog incompleteness will continue to be a problem, especially as new line searches move into the submillimeter region where laboratory observations are sparse, and an essential part of future programs must be continued close ties and access to state-of-the-art laboratory spectrometers. The improved sensitivity of the new telescopes will give roughly a factor of ten increase in speed, and will enable the chemical composition of many interstellar objects to be determined for the first time.

\section{The Caltech OVRO $1.3 \mathrm{~mm}$ Survey of OMC-1}

A major goal of such surveys will be an assessment of the influence of the chemistry which occurs in diffuse and dense molecular clouds on the formation of stars and planets. That is to say, does the star formation process completely erase the chemical history of matter used to build, for example, the solar system, or is memory significant? The Orion-Monoceros complex, of which OMC-1 is but a small part, lies only $500 \mathrm{pc}$ from the sun. Due to its proximity and brightness, it has become the prototypical star formation region. Here, we use the studies of this cloud to outline the tremendous chemical heterogeneity of star forming clouds and the impact of this heterogeneity on the evolution of early bodies in the solar system. A more complete description of the OVRO linesearch and its chemical implications has been presented by Blake et al. ${ }^{20}$

The cool and extended gas of the Orion-Monoceros complex is visible in observations of OMC-1 as a strip or "ridge" of material running roughly N-S. A velocity gradient exists across the cloud, with the southern section appearing at $8 \mathrm{~km} \mathrm{~s}^{-1}$ and the northern section appearing at $10 \mathrm{~km} \mathrm{~s}^{-1}$. In the center of the cloud several compact sources dominate the spectral appearance of this source in both continuum and spectral line observations, associated primarily with IRc 2; a young, massive star which is in the process of disrupting the cloud core. Most prominent among these sources is the "plateau" feature, so named for the broad lines which emanate from this region. First observed as very high velocity wings of $\mathrm{CO}^{21}$, this source has since been observed in many molecules and seems to possess two distinct signatures. The first is a high velocity outflow with speeds of up to $100 \mathrm{~km} \mathrm{~s}^{-1}$, and the second is a "low" velocity outflow with linewidths closer to $20 \mathrm{~km} \mathrm{~s}^{-1}$.

The supersonic velocities in the plateau gas drive shocks into the surrounding medium, compressing and heating the gas. A particularly interesting region of this type in OMC-1 has been labelled the "compact ridge" source, and lies slightly south of IRc 2 on the edge of the $8 \mathrm{~km} \mathrm{~s}^{-1}$ ridge cloud. As its name implies, it is unresolved in single dish maps. It is slightly warmer than the extended ridge (100-150 $\mathrm{K}$ versus $40-50 \mathrm{~K})$ and it also has a noticeably different chemistry. Closer to IRc 2 itself is a large clump, or set of clumps, known as the"hot core", 
with temperatures and densities approaching $300 \mathrm{~K}$ and $10^{8} \mathrm{~cm}^{-3}$ ! Unlike either the plateau or compact ridge sources, it is heated passively by radiation from the young star(s). Differences in the spectral line profiles of these sources must be used to deconvolve their individual contributions in single dish surveys of OMC-1 because they are often superposed along the line of sight. With recently developed millimeter wave interferometers, however, it has been possible to examine the chemical variations within this surprising source at high angular resolution.

Many different models of the Orion cloud core have been presented, but all boil down to essentially this: Outflow from IRc 2 creates directly the low and high velocity plateau sources. Where these winds strike the surrounding cloud shocks are created. In the shock front themselves the temperatures are very high, but regions close to and not in direct contact with the shock will be heated and compressed (the compact ridge). The high velocity plateau is formed along the most rapid density gradients in the cloud, while the low velocity plateau is created where the outflowing wind strikes the densest parts of the surrounding medium. Material left over from the formation of IRc 2 but not yet disrupted by the outflow is observed as the hot core. We now examine the chemical content of these four regions, starting first with the extended ridge clouds.

Table 2

\begin{tabular}{|c|c|c|c|c|c|}
\hline \multirow[b]{3}{*}{ SPECIES } & \multicolumn{5}{|c|}{ AbUndanCE Relative to $\mathrm{H}_{2}$} \\
\hline & \multirow[b]{2}{*}{ OMC-1" } & \multirow[b]{2}{*}{$\mathrm{Sgr} \mathrm{B}^{\mathrm{b}}$} & \multirow[b]{2}{*}{ TMC $-1^{\mathrm{C}}$} & \multicolumn{2}{|c|}{ Herbst and Leung ${ }^{d}$} \\
\hline & & & & Model $1^{\circ}$ & Model $2^{r}$ \\
\hline $\mathrm{CO}$ & $5.0 \times 10^{-5}$ & $6.0 \times 10^{-3}$ & $5.8 \times 10^{-5}$ & $1.5 \times 10^{-4}$ & $3.4 \times 10^{-4}$ \\
\hline $\mathrm{CN}$ & $3.3 \times 10^{-9}$ & $3.4 \times 10^{-10}$ & $3.0 \times 10^{-8}$ & $3.2 \times 10^{-9}$ & $2.4 \times 10^{-5}$ \\
\hline CS $\ldots \ldots \ldots \ldots \ldots \ldots$ & $2.5 \times 10^{-9}$ & $3.0 \times 10^{-9}$ & $2.0 \times 10^{-9}$ & $4.8 \times 10^{-8}$ & $1.5 \times 10^{-7}$ \\
\hline SO ................ & $\leqslant 9.3 \times 10^{-10}$ & $1.5 \times 10^{-10}$ & $5.0 \times 10^{-9}$ & $1.2 \times 10^{-8}$ & $4.5 \times 10^{-11}$ \\
\hline $\mathrm{SO}_{2} \ldots \ldots \ldots \ldots \ldots$ & $<3.3 \times 10^{-9}$ & $2.0 \times 10^{-9}$ & $\ldots$ & $4.8 \times 10^{-8}$ & $1.3 \times 10^{-12}$ \\
\hline $\mathrm{C}_{2} \mathrm{H} \ldots \ldots \ldots \ldots \ldots$ & $5.3 \times 10^{-9}$ & … & $8.4 \times 10^{-9}$ & $2.4 \times 10^{-10}$ & $2.7 \times 10^{-6}$ \\
\hline $\mathrm{HCH} \ldots \ldots \ldots \ldots \ldots$ & $5.0 \times 10^{-9}$ & $-3.0 \times 10^{-9}$ & $1.2 \times 10^{-8}$ & $5.5 \times 10^{-9}$ & $1.0 \times 10^{-6}$ \\
\hline HNC $\ldots \ldots \ldots \ldots . . .$. & $5.3 \times 10^{-10}$ & $\sim 3.0 \times 10^{-9}$ & $8.0 \times 10^{-9}$ & $5.5 \times 10^{-9}$ & $1.0 \times 10^{-6}$ \\
\hline $\mathrm{HCO}^{+} \ldots \ldots \ldots \ldots$ & $2.3 \times 10^{-9}$ & $2.3 \times 10^{-9}$ & $8.0 \times 10^{-9}$ & $1.0 \times 10^{-8}$ & $7.3 \times 10^{-9}$ \\
\hline $\mathrm{HCS}^{+} \ldots \ldots \ldots \ldots$ & $5.3 \times 10^{-11}$ & $3.0 \times 10^{-11}$ & $5.0 \times 10^{-10}$ & $1.4 \times 10^{-11}$ & $5.3 \times 10^{-11}$ \\
\hline $\mathrm{H}_{2} \mathrm{D}+\ldots \ldots \ldots$ & $-1.0 \times 10^{-10}$ & ... & $<3.0 \times 10^{-8}$ & ... & $-1.1 \times 10^{-10}$ \\
\hline $\mathrm{HC}_{3} \mathrm{~N} \ldots \ldots \ldots \ldots$ & $1.3 \times 10^{-10}$ & $3.8 \times 10^{-10}$ & $6.0 \times 10^{-9}$ & $3.5 \times 10^{-12}$ & $9.2 \times 10^{-8}$ \\
\hline $\mathrm{HC}_{5} \mathrm{~N} \ldots \ldots \ldots \ldots$ & $\leqslant 2.3 \times 10^{-11}$ & $\cdots$ & $1.0 \times 10^{-8}$ & $8.9 \times 10^{-16}$ & $6.2 \times 10^{-10}$ \\
\hline $\mathrm{CH}_{3} \mathrm{CN} \ldots \ldots \ldots$ & $3.2 \times 10^{-10}$ & $2.0 \times 10^{-10}$ & $5.0 \times 10^{-10}$ & $1.5 \times 10^{-11}$ & $8.5 \times 10^{-8}$ \\
\hline $\mathrm{H}_{2} \mathrm{CO} \ldots \ldots \ldots$ & $1.7 \times 10^{-8}$ & $3.0 \times 10^{-9}$ & $1.2 \times 10^{-8}$ & $7.7 \times 10^{-9}$ & $5.7 \times 10^{-8}$ \\
\hline $\mathrm{CH}_{3} \mathrm{OH} \ldots \ldots \ldots \ldots$ & $1.2 \times 10^{-7}$ & $2.0 \times 10^{-8}$ & $\ldots$ & $5.6 \times 10^{-10}$ & $1.6 \times 10^{-8}$ \\
\hline
\end{tabular}

- Derived from this work and Johansson et al. 1984.

- Derived from Cummins, Linke, and Thaddeus 1986.

- From the data compilation in Leung. Herbst. and Huebner 1984.

¿ 1986.

- Model results for $\mathrm{C} / \mathrm{O}=0.4, n_{\mathrm{H}_{2}}=10^{5} \mathrm{~cm}^{-3}, t=10^{7} \mathrm{yr}$.

Model results for $\mathrm{C} / \mathrm{O}=1.3, n_{\mathrm{H}_{2}}=10^{5} \mathrm{~cm}^{-3}, t=10^{7} \mathrm{yr}$.

- Abundances derived by Phillips et al. 1985.

Early observations of simple species such as $\mathrm{H}_{2} \mathrm{CO}$ and $\mathrm{HCN}$ in molecular clouds showed a strong correlation of abundance with density, an effect ascribed to the depletion of molecules onto grain mantles. A major result of more recent observations, especially the accurately calibrated spectral line surveys from Onsala, OVRO, and Bell Labs, is that the abundances of simple species appear to be rather constant not only in dense clouds, but in other sources as well. Table 2 presents a listing of some of the more abundant molecules in the Orion, Sagittarius, and Taurus molecular 
clouds in addition to their predicted abundances in the gas phase model of Herbst and Leung ${ }^{22}$. The two predictions refer to gas phase $\mathrm{C} / \mathrm{O}$ ratios of 0.4 (solar) and 1.3. As may be seen, the abundances of species like $\mathrm{CO}, \mathrm{CS}$, and $\mathrm{HCO}^{+}$are roughly constant, and their abundance is well predicted by the gas phase model.

To a great extent, the agreement between observation and gas phase theory must be considered coincidental. For example, many of the reactions used in the model have not been measured experimentally; and as we have noted earlier gas-grain interactions have not been taken into account. The lack of abundance correlations with density does suggest, however, that for many species gas phase processes dominate their chemistry and also that an efficient grain desorption mechanism must exist which either does not significantly chemically process material or which produces species similar to those inferred from ion-molecule reaction networks.

The most glaring deficiencies in the model involve atomic carbon and related molecular species such as $\mathrm{CCH}$ and the polyacetylenes. This problem is especially severe in cold clouds like TMC-1, as Table 2 demonstrates. At steady state most gas phase models predict little C I or other carbon rich radicals because reactions like

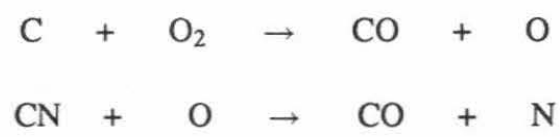

convert most of these species into more stable forms $\left(\mathrm{O}_{2}\right.$ is predicted to be a major oxygen reservoir). Thus, for molecular species other than $\mathrm{CO}$ these models predict that the observable $\mathrm{C}$ and $\mathrm{O}$ inventories should reflect the total $\mathrm{C} / \mathrm{O}$ ratio. In the extended Orion ridge clouds this ratio is currently near one, if $\mathrm{C} \mathrm{I}$ is included the ratio exceeds ten. Thus, of all the species studied to date, C I shows the greatest discrepancy between theory and observation; and several mechanisms have therefore been brought forward to explain the high observed abundance. The major point here is that although most of the abundances of simple closed shell species are well predicted by gas phase models, the abundances of reactive hydrocarbon radicals are not. A related problem, discussed in more detail later, is the high abundances of complex species like $\mathrm{CH}_{3} \mathrm{OH}$ and $\mathrm{HCOOCH}_{3}$ also observed in clouds like OMC-1. The basic question is how clouds can simultaneously be rich in reactive carbon rich radicals like $\mathrm{CCH}$ and also in stable oxygen containing species like $\mathrm{HCOOCH}_{3}$. As might be expected, the answer most likely lies in the complex physical variations of molecular clouds along a given line of sight and the finite spatial resolution that characterizes any observational study of dense cloud chemistry.

For example, outflowing gas from IRc 2 lies along the same line of sight as much of the surrounding quiescent cloud, and signals from each region will be detected simultaneously by millimeter-wave telescopes. Whereas gas in the exterior regions appears to be controlled primarily by ion-molecule chemistry and depletion effects, the chemical composition of the plateau material is dominated by high temperature chemistry induced by shocks. A graphical demonstration of the differences between the quiescent ridge chemistry and that of the plateau source is depicted in Figure 4. The most striking result is the tremendous drop in the abundances of carbon rich species like $\mathrm{C}, \mathrm{CCH}$, and $\mathrm{CN}$ and the concomitant rise in the abundance of species such as $\mathrm{H}_{2} \mathrm{O}, \mathrm{HCN}$, $\mathrm{SiO}, \mathrm{H}_{2} \mathrm{~S}$, and $\mathrm{SO}_{2}$. While the extended ridge clouds appear observationally to have a deficit of oxygen in the gas phase, the plateau gas seems to have a gas phase $\mathrm{C} / \mathrm{O}$ ratio that is solar or lower. Indeed, the observed value for molecules other than $\mathrm{CO}$ is $\mathrm{C} / \mathrm{O} \leq 0.5$ not including water (observed as HDO), and $<<1$ for all reasonable estimates of the residual deuterium fractionation in HDO. The higher oxygen fugacity and the very high temperatures behind the shock front 
drive most of the carbon into stable forms such as $\mathrm{CO}$, but also enhance the abundances of more refractory species such as sulfur and silicon via grain disruption or evaporation.

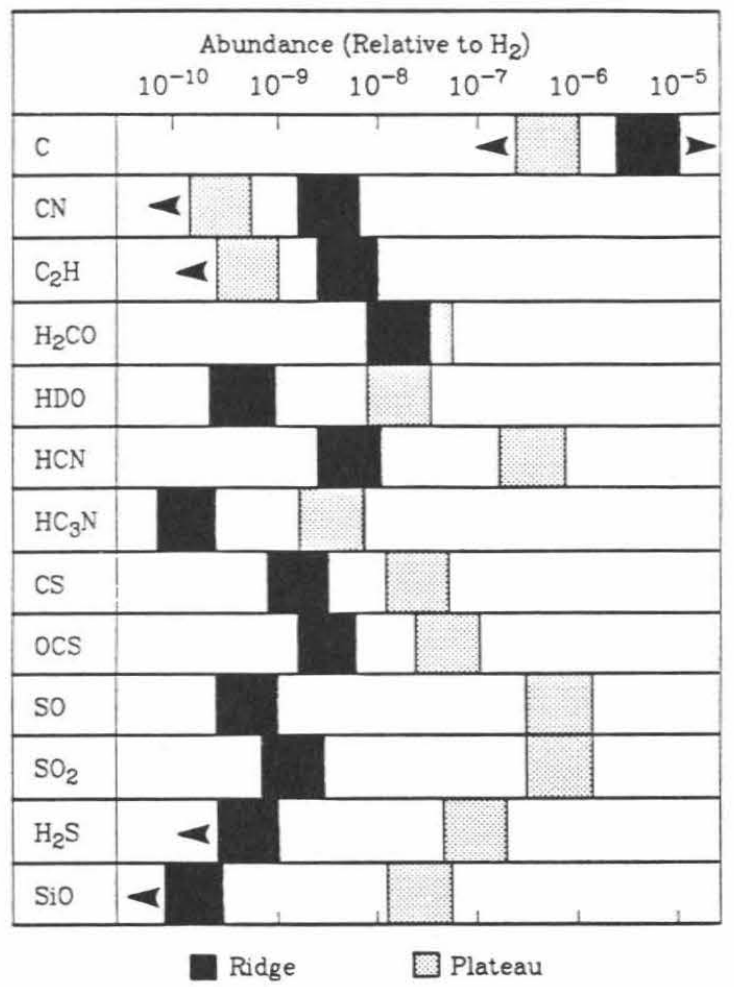

Figure 4.- Graphical comparison of the OMC-1 ridge and plateau fractional abundances.

High temperature chemistry is also evident in observations of the hot core, but with a considerably different signature. Here the critical importance of gas-grain interactions become apparent as is shown graphically in Figure 5. Again, the abundances of reactive, carbon rich species like $\mathrm{C}, \mathrm{CCH}$, and $\mathrm{CN}$ are significantly reduced, with the observed $\mathrm{C} / \mathrm{O}$ ratio in molecular form less than unity (as in the plateau gas the actual ratio depends sensitively on the assumed deuterium fractionation in HDO). Because the heating is passive, however, grain vaporization does not occur and the abundances of the refractory sulfur and silicon species remain low. The high densities and temperatures in the hot core source shut down ion-molecule networks, and are sufficient to release much of the material stored over the cloud lifetime in grain mantles. Indeed, the composition of the hot core is most consistent with chemistry that is dominated by kinetic equilibrium and evaporation from grains. For example, water and ammonia are observed to be tremendously enhanced in the hot core to levels near that observed in near infrared absorption bands of water ice mantles on interstellar grains. The water observations are especially noteworthy as they are carried out using HDO, which seems to have retained significant deuterium fractionation even though the source kinetic temperature is well above $100 \mathrm{~K}$. The most likely explanation is therefore that the observed HDO is "fossil" water trapped onto grains throughout 
the cloud lifetime and released when star formation has raised the grain temperatures sufficiently to evaporate their icy mantles ${ }^{25}$. Further, the conversion of polyacetylenes into more hydrogenated species like $\mathrm{HC}_{3} \mathrm{~N} \rightarrow \mathrm{C}_{2} \mathrm{H}_{5} \mathrm{CN}$ must occur on grains because reactions such as

$$
\mathrm{HC}_{3} \mathrm{NH}^{+}+\mathrm{H}_{2} \rightarrow \mathrm{C}_{2} \mathrm{H}_{3} \mathrm{CN}^{+}+\mathrm{H} \text { etc. }
$$

are highly endothermic, while hydrogenation on surfaces is known to be fairly efficient, at least in the laboratory.

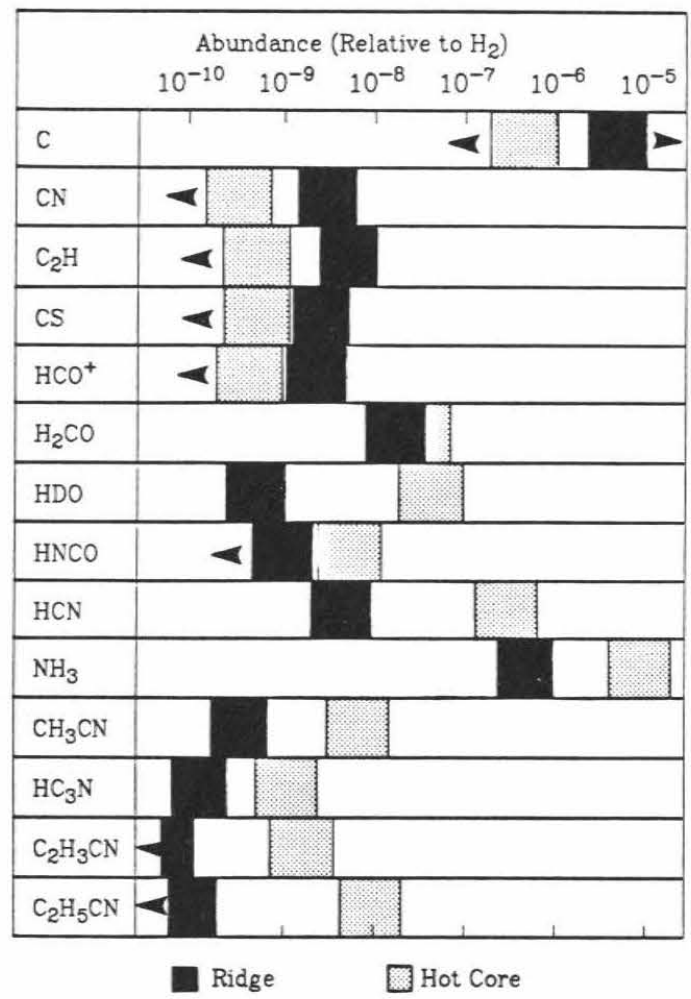

Figure 5.- Graphical comparison of the OMC-1 ridge and hot core abundances.

Finally, the importance of mixing in active cores like that in OMC-1 is demonstrated by the composition of the compact ridge source. It is here that the abundances of complex oxygen containing organics such as $\mathrm{CH}_{3} \mathrm{OH}$ and $\mathrm{HCOOCH}_{3}$ peak. These species could either be produced by grain mantle hydrogenation of $\mathrm{CO}$, etc., or by gas phase routes. A possible gas phase scenario is presented in Figure 6 which relies heavily on radiative association reactions of the type originally outlined by Herbst ${ }^{1}$. Theoretical predictions of the rates of such reactions, tested experimentally only for the $\mathrm{CH}_{3}{ }^{+}+\mathrm{H}_{2}$ system ${ }^{26}$, indicate that they can become quite fast for large complexes - with some reactions occurring at the collision rate even in the presence of exothermic bimolecular channels! In this model, mixing of oxygen and water rich gas from the plateau with ion rich gas from the extended clouds is predicted to form the ionic precursor to methanol and methanol itself via the reactions 


$$
\begin{aligned}
& \mathrm{CH}_{3}^{+}+\mathrm{H}_{2} \mathrm{O} \rightarrow \mathrm{CH}_{3} \mathrm{OH}_{2}^{+}+\mathrm{h} \nu \\
& \mathrm{CH}_{3} \mathrm{OH}_{2}^{+}+\mathrm{e}^{-} \rightarrow \mathrm{CH}_{3} \mathrm{OH}+\mathrm{H} .
\end{aligned}
$$

The resulting products then synthesize a variety of isomerically specific molecules such as dimethyl ether and methyl formate through reactions with other hydrocarbons. Larger species should also be formed by such networks, but their abundances will lie below that detectable with current instrumentation. The resulting $\mathrm{C} / \mathrm{O}$ ratio in these larger species is near unity.

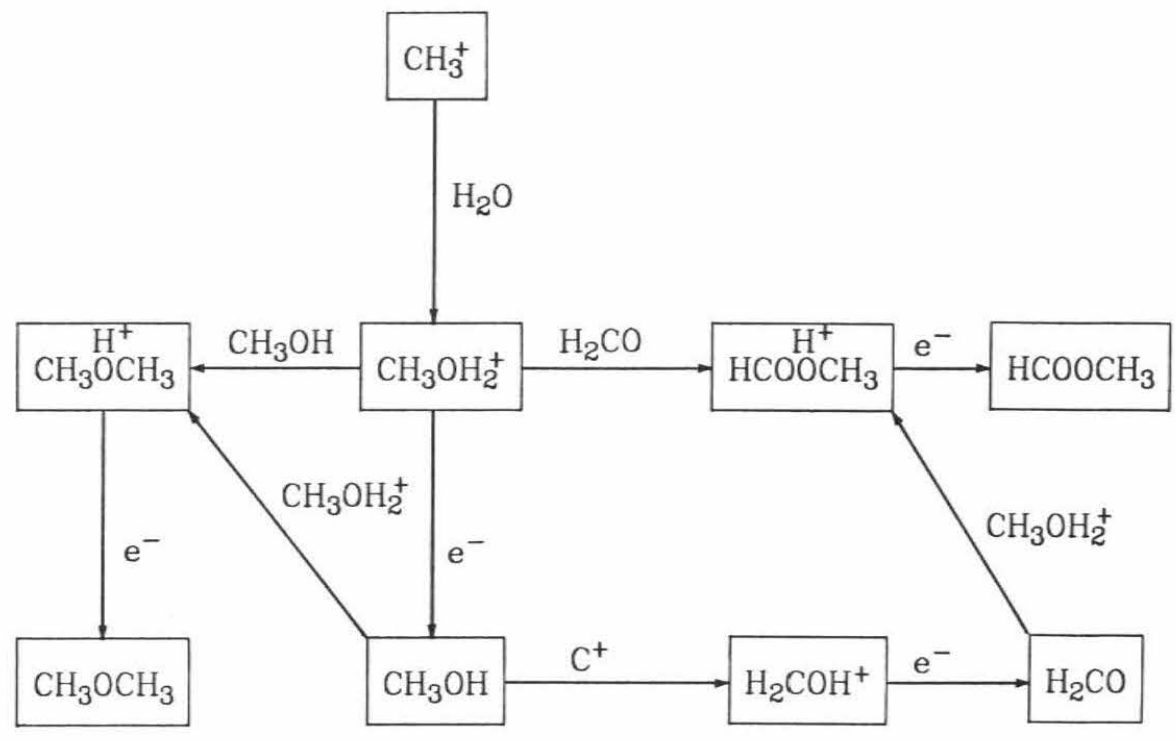

Figure 6.- Schematic outline of the proposed ion-molecule network responsible for the formation of complex molecules in the OMC-1 compact ridge.

A major point in favor of the gas phase model versus grain mantle synthesis is the observational selectiveness of interstellar chemistry. Namely, dimethyl ether and methyl formate are found to be much more abundant than their isomers ethanol and acetic acid. A simple steady state solution to the chemistry outlined in Figure 6 reproduces the observed abundances in OMC- 1 to within a factor of a few, and, while most likely fortuitous, is probably indicative of the dominance of a gas phase synthesis of these molecules.

Further evidence for the gas phase scheme is provided by recent high angular resolution observations of methanol in OMC-1 by Plambeck and Wright ${ }^{27}$ (see contribution by R. Plambeck). If methanol and the other related species were produced primarily by grain mantle catalysis, then they should show observational correlation with other grain mantle products. For example, ammonia, ethyl cyanide and water show striking observational similarities in Orion. Methanol, however, appears to be anticorrelated with these species. These and other observations are most consistent with a general model of active cloud cores in which the surrounding, cooler medium contains the bulk of the observed reactive hydrocarbons while the cloud cores have liberated the more refractory elements and driven the chemistry towards kinetic equilibrium. 


\section{STUDIES OF THE CHEMICAL EVOLUTION OF PROTOSTARS}

The Orion cloud core is an example of massive star formation, and a natural question is just how applicable the results from high mass sources are to the formation of lower mass stars like our own sun. In massive star forming clouds, clustering appears to be common, resulting in significant alteration of the overall cloud chemistry. It is generally accepted that, as single low mass stars condense out of molecular clouds, the circumstellar gas must eventually become flattened so that the remnant cloud around the protostellar object acquires a disk-like morphology, and that the gas surrounding the protostar need only represent a small fraction of the total cloud mass. With the advent of interferometric techniques at millimeter and infrared wavelengths it has recently become possible to identify such disks directly. For example, around the objects HL Tauri, L1551 IRS5 and IRAS 16293-2422 are extended, flattened structures with radii of order a few hundred A.U. ${ }^{28,29}$. Because of the parallels with our own solar system, it is important to investigate the chemistry and dynamics of such proto-planetary disks in detail. While dynamical studies ultimately require the resources of instruments capable of higher resolution, single telescopes can explore the chemical nature of these sources. Broadband surveys such as those outlined above may no longer be possible due to sensitivity limitations, but deep searches for selected species should give decent estimates of the chemical environment of circumstellar material.

Indeed, the necessity for chemical analyses of the immediate circumstellar environment of young stellar objects and protostars has already been highlighted by the case of IRAS 162932422. OVRO maps of this object in the lines of $\mathrm{CO}$ and its isotopes and VLA maps in $\mathrm{NH}_{3}$, although at similar resolution, are markedly different ${ }^{29}$. The OVRO $\mathrm{C}^{18} \mathrm{O}$ and $2.7 \mathrm{~mm}$ continuum measurements suggest a disk of size $1800 \mathrm{x} \leq 800 \mathrm{~A}$.U. This disk is aligned perpendicular to the direction of a high velocity molecular outflow and to the magnetic field. The $\mathrm{NH}_{3}$ maps show an elongated structure, with similar orientation, but considerably greater in extent, 7200 A.U. The $\mathrm{NH}_{3}$ is obviously tracing different gas than the $\mathrm{C}^{18} \mathrm{O}$, but it is not clear without further chemical analyses precisely how to characterize the relevant properties of the disk.

Numerical simulations of such disk-like structures have now determined the likely temperature and density gradients in these objects. One finds that only in the inner few A.U. of the disk do temperatures climb high enough to vaporize the dust. At the distances of the major planets the temperatures have dropped sufficiently low that degassing of even the grain mantles becomes unlikely in the bulk gas. Models of the transport properties of accretion disks show that it is very difficult to move matter from large radii into smaller radii and back out again. The major result is thus that it is kinetically impossible to transform stable species such as $\mathrm{CO}$ and $\mathrm{N}_{2}$, the dominant interstellar forms of carbon and nitrogen, into complex hydrocarbons via homogeneous gas phase chemistry at radii beyond 5-10 A.U. As such, one expects considerable persistence of memory in these objects, at least in the outer portion of the disk where major planets and comets form. The observations of significant fractionation effects in meteoritical and cometary particles as well as in planetary atmospheres may well be due to the preservation of primarily interstellar material throughout the collapse phase.

Several processes will act to alter the composition from a pristine interstellar signature, however. Most of these effects are similar to those operating in massive molecular cloud cores, but on a much smaller scale. For example, as matter rains onto the disk from the surrounding molecular cloud, it experiences a discontinuous velocity change at the disk. This so called accretion shock front processes all of the infalling material through the high temperature environment of a roughly $10 \mathrm{~km} \mathrm{~s}^{-1}$ shock, although the effect drops sharply at larger radii. The investigation of shock chemistry and sputtering effects in this regime is therefore of the utmost importance. 
Secondly, although it is true that homogeneous gas phase conversion of $\mathrm{CO}$ and $\mathrm{N}_{2}$ into organics is prohibited in the bulk gas, in the regions around large gaseous protoplanets the increased temperatures and densities may be sufficient to drive such conversions ${ }^{30}$. As in the Orion hot core region, the high densities and temperatures in protoplanetary sub-nebulae reduce the effectiveness of ion-molecule chemistry, and enable new neutral-neutral pathways to emerge. Clustering in the gas phase increases rapidly as large protoplanets form, reflecting the significance of many body effects. Surface catalysis will also become considerably more active in such environments.

Since comets have been hypothesized to arise from protoplanetary regions near the current orbits of Uranus and Neptune, the alteration of protosolar gas and dust during planetary and cometary formation must be addressed in the future. Ideally, one would ultimately like to construct a kinetic model incorporating dynamical effects and heterogeneous catalysis in a realistic fashion. Before this can occur, however, increasingly rigorous theoretical calculations on the dynamics of the accretion process and experimental work on shock chemistry, nucleation, and surface catalysis must be performed.

\section{CONCLUSIONS}

A tremendous amount of both observational and theoretical progress has been made in the analysis of interstellar chemistry since the discovery of $\mathrm{OH}$ in dense clouds some twenty-five years ago. Plausible formation mechanisms in the gas phase and on grain surfaces now exist for most of the simple observed species, and models of their abundances can be performed. Observational work has given us not only estimates of the chemical composition of objects ranging from diffuse interstellar clouds to the extended atmospheres of evolved stars, but also significant constraints on the physical conditions there. Studies with the FCRAO 14 meter millimeter-wave telescope have contributed substantially to our understanding of all of these objects, and will continue to do so in the future with its improved focal plane instrumentation and enclosure. While the general picture of interstellar chemistry is fairly clear at this point, for further progress to be made much more detailed observations and calculations will be required. In particular, the evolution of molecular complexity in dense clouds and its possible impact on the composition and properties of solar system objects ranging from meteorites and comets to planetary atmospheres remain obscure.

Observationally, we are now in a position to examine directly molecular cloud cores similar to that which formed the solar system. It will therefore be possible to probe, for the first time, changes which occur in the gas phase speciation of matter associated with the star formation process. In the laboratory, state-of-the-art experiments in laser spectroscopy and in surface science are in a position to examine processes directly relevant to the formation of gas and dust in the interstellar medium and their assembly into stars and planets. Future theoretical work on interstellar chemistry with supercomputers should be able to include heterogeneous effects such as dynamics and catalysis in a realistic manner. Only through a combination of such efforts will the true details of our origins be revealed. 


\section{REFERENCES}

[1] Herbst, E. 1987, Interstellar Processes, Hollenbach, D.J. and Thronson, H.A., Jr. eds., D. Reidel Publ., 611.

[2] Prasad, S.S. et al. 1987, Interstellar Processes, Hollenbach, D.J. and Thronson, H.A., Jr. eds., D. Reidel Publ., 631.

[3] Irvine, W.M., Goldsmith, P.F., and Hjalmarson, A. 1987, Interstellar Processes, Hollenbach, D.J. and Thronson, H.A., Jr. eds., D. Reidel Publ., 561.

[4] Spitzer, L., Jr. 1978, Physical Processes in the Interstellar Medium, Wiley and Sons, Inc.

[5] Gas Phase Ion Chemistry, M.T. Bowers, ed., 1979, Academic Press.

[6] Clary, D.C. 1985, Mol. Phys. 54, 605.

[7] Boland, W. and de Jong, T. 1984, Astr. Ap. 134, 87.

[8] Tarafdar, S.P. et al. 1985, Ap. J. 289, 220.

[9] Prasad, S.S. and Huntress, W.T., Jr. 1980, Ap. J. Suppl. 43, 1.

[10] Hagen, W., Tielens, A.G.G.M., and Greenburg, J.M. 1983, Astr. Ap. 117, 132.

[11] Greenburg, J.M. 1983, Workshop on Laboratory and Observational Infrared Spectra of Interstellar Dust, Wolstencroft, R.D. and Greenburg, J.M., eds., Royal Observatory of Edinburgh Publ., 1.

[12] Gillet, F.C. and Forrest, W.J. 1973, Ap. J. 179, 483.

[13] Williams, D.A. and Hartquist, T.W. 1984, Mon. Not. Roy. Astr. Soc. 210, 41.

[14] Leger, A., Jura, M., and Omont, A. 1985, Astr. Ap. 144, 147.

[15] d'Hendecourt, L.B., Allamandolla, L.J., and Greenburg, J.M. 1985, Astr. Ap. 152, 130.

[16] Johansson, L.E.B. et al. 1984, Astr. Ap. 130, 227.

[17] Cummins, S.E., Linke, R.A., and Thaddeus, P. 1986, Ap. J. Suppl. 60, 819.

[18] Sutton, E.C. et al. 1984, Ap. J. Suppl. 58, 341.

[19] Blake, G.A. et al. 1986, Ap. J. Suppl. 60, 357.

[20] Blake, G.A. et al. 1987, Ap. J. 315, 621.

[21] Wilson, R.W., Jefferts, K.B., and Penzias, A.A. 1970, Ap. J.(Letters) 161, L43.

[22] Herbst, E. and Leung, C.M. 1986, Ap. J. 310, 378.

[23] Leung, C.M., Herbst, E., and Huebner, W.F. 1984, Ap. J. Suppl. 56, 231.

[24] Phillips, T.G. et al. 1985, Ap. J.(Letters) 294, L45.

[25] Plambeck, R.L. and Wright, M.C.H. 1987, Ap. J.(Letters) 317, L101.

[26] Barlow, S.E., Dunn, G.H., and Schauer 1984, K., Phys. Rev. Lett. 52, 902.

[27] Plambeck, R.L. and Wright, M.C.H. 1988, Ap. J.(Letters), submitted.

[28] Sargent, A.I. and Beckwith, S. 1986, Ap. J. 323, 294.

[29] Mundy, L.G., Wilking, B.A., and Myers, S. 1986, Ap. J.(Letters) 311, L75-L79.

[30] Prinn, R.G. and Fegley, B. 1988, Origin and Evolution of Atmospheres, University of Arizona Press, in press. 